\title{
UPGRADE OF THE TRIGGER SYSTEM OF
}

THE ATLAS LIQUID ARGON CALORIMETERS

\section{INTRODUCTION}

ATLAS is a general-purpose detector in particle physics, designed to study proton-proton collisions produced at the Large Hadron Collider (LHC). A series of upgrade programs are foreseen in next 10 years to explore the full physics potential of the LHC experiments. The ATLAS Liquid argon (LAr) sampling calorimeters produce a total of 182,486 signals, which are digitized and processed by the front-end and back-end electronics for each triggered event. In addition, the front-end electronics provides the signals to the first level trigger system. In 2020, instantaneous luminosities of $(2-3) \times 10^{34} \mathrm{~cm}^{-2} \mathrm{~s}^{-1}$ are expected, far beyond that for which the detector was designed. The new trigger strategy and electronic upgrades are required to use the full potential of the ATLAS detector.

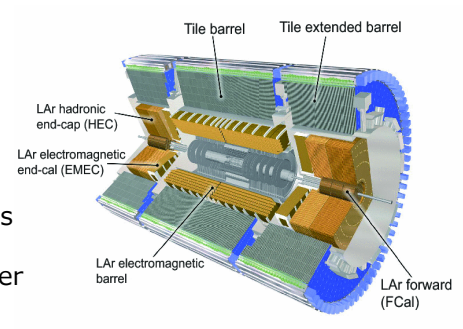

\section{TRIGGER STRATEGY}

The Level-1(L1) trigger rate is limited by the readout bandwidth of the front-end detector electronics to about $100 \mathrm{kHz}$, and the latency of the L1 trigger is limited by the depth of the front-end detector pipeline memory up to $2.5 \mu \mathrm{s}$. The strategy of the L1 calorimeter trigger upgrade for Phase-I is to use digital, finer granularity signals from the LAr electromagnetic calorimeter (Super-Cell), to run more effective algorithms using smart variables. Retaining the low transverse energy threshold $\left(E_{T}\right)$ by improving electron/photon separation from jets.
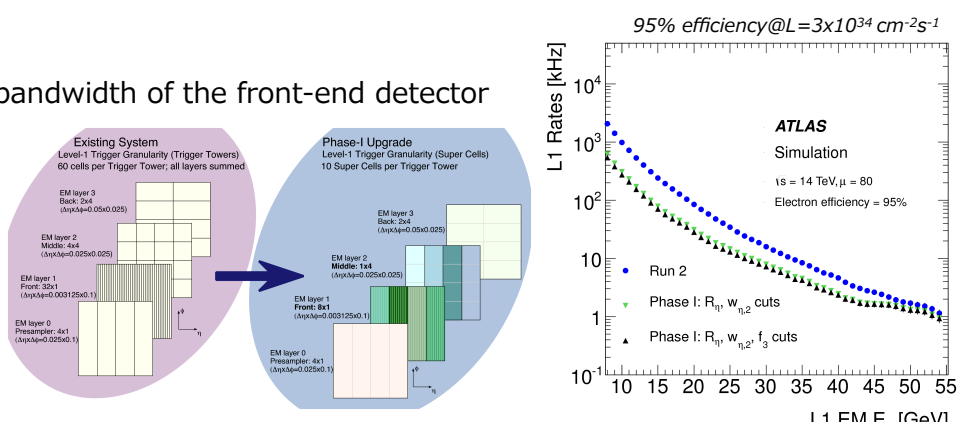

L1 EM E $E_{\mathrm{T}}[\mathrm{GeV}]$

\section{NEW READOUT ARCHITECTURE}

The use of the scheme based on Super-Cells requires a replacement of the Layer Sum Boards (LSBs) for the front and middle layers $\longrightarrow$ New boards/backplanes replacement

LAr Trigger Digitization Board (LTDB, on-detector)

- Build analog sums as input for TBBs (tower builder board)

- Digitize Super-Cell analog signals for LDPS (digitization board)

Radiation tolerance 100Gy*, latency 275ns, low power consumption

LAr Digitization Processing System (LDPS, off-detector)

- Receive the digital signals (from 124 LTDBs, 34,000 Super-Cells)

- Extract calibrated energy from raw ADC sampling data by applying a digital filtering technique (FPGA-base)

Reception/transmission 25/41 [Tb/s], latency 350ns

L1Calo trigger system

* phase-II upgrade for $H L-L H C$ run, $\int L=3 a b^{-1}$

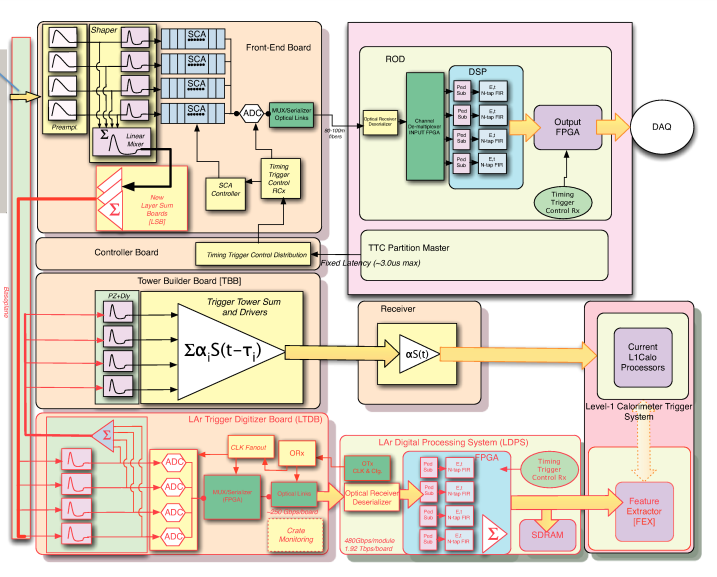

\section{R\&D STUDIES FOR THE ELECTRONICS UPGRADES}

\section{$\checkmark$ Mixed-signal Front-end ASICs}

- Digitization speed $\geq 40$ MSPS

- Resolution (ENOB) $\geq 11$

Both commercial and custom ADC chips are options.

COTS TI-ADS5281 : radiation torelant survived after $\sim 89 \mathrm{kGy}$ irradiation Custom ASIC ADCs: based on IBM CMOS8RF (130nm) technology for low latency and low power consumption.

- 4-stage SAR-based pipeline ADC (<50mw)

- Full SAR-based architecture ADC (<10mW @20MSPS tested)

\section{High-speed/high density digital} processing system (ATCA platform)

- 54 high-speed transceivers (5.12Tb/ps for Rx,10.5Tb/s for Tx) - Sufficient DSP processing power

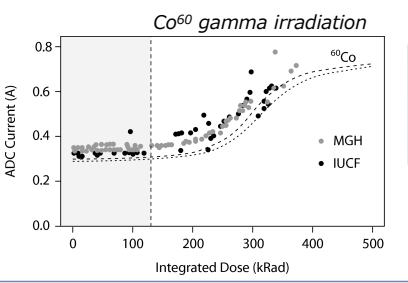

Two hi-end commercial FPGAs, Altera and Xilinx families are used for the development.

\begin{tabular}{c|c|c|c|} 
Resource & $\begin{array}{c}\text { Estimated } \\
\text { Requirement }\end{array}$ & Specification \\
\cline { 2 - 4 } & FPGA-I & FPGA-II \\
\hline
\end{tabular}

\begin{tabular}{|c|c|c|c|c|}
\hline Resource & & & & \\
\hline Register $\left[10^{3}\right.$ & Requirement & FPGA-I & FPGA-II & \\
\hline LUT $\left[10^{-}\right.$ & $\begin{array}{l}184 \\
120\end{array}$ & $\begin{array}{l}14<4 \\
712\end{array}$ & $\begin{array}{l}1300 \\
900\end{array}$ & FPGA-I \\
\hline TX/RX & 54 & 96 & 96 & Xilinx Virtex-7 \\
\hline DSP & 778 & $3360\left(^{*}\right)$ & $1518\left(^{*}\right)$ & FPGA-II \\
\hline lockRam [Mbit] & 13.5 & 677 & 57.0 & Altera $A R$ \\
\hline
\end{tabular}
BlockRam [Mbit]
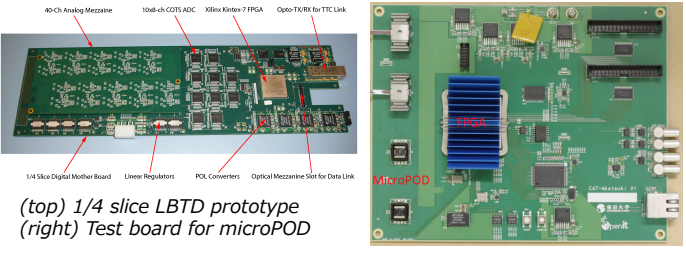

\section{SUMMARY}

An upgrade of the trigger readout for the ATLAS LAr calorimeters is essential for running in high luminosity and pileup conditions. The improved spatial granularity of the trigger primitives reduces the trigger rate. The R\&D studies are ongoing towards Phase-I (II) upgrades. The upgrade designs have been validated. The full system test of the upgrade electronics are going smoothly at CERN, and in-situ demonstrator will be foreseen for the run starting in 2015.

\section{REFERENCES}

[1] The ATLAS Collaboration, "ATLAS Liquid Argon Calorimeter Phase-I upgrade Technical Design Report", CERN-LHCC-2013-017 